\title{
Orthogonality and minimality in the homology of locally finite graphs
}

\author{
Reinhard Diestel \\ Department of Mathematics \\ University Hamburg \\ Hamburg, Germany
}

\author{
Julian Pott \\ Department of Mathematics \\ University Hamburg \\ Hamburg, Germany
}

Submitted: Oct 25, 2013; Accepted: Aug 29, 2014; Published: Sep 11, 2014

Mathematics Subject Classification: 05C

\begin{abstract}
Given a finite set $E$, a subset $D \subseteq E$ (viewed as a function $E \rightarrow \mathbb{F}_{2}$ ) is orthogonal to a given subspace $\mathcal{F}$ of the $\mathbb{F}_{2}$-vector space of functions $E \rightarrow \mathbb{F}_{2}$ as soon as $D$ is orthogonal to every $\subseteq$-minimal element of $\mathcal{F}$. This fails in general when $E$ is infinite.

However, we prove the above statement for the six subspaces $\mathcal{F}$ of the edge space of any 3-connected locally finite graph that are relevant to its homology: the topological, algebraic, and finite cycle and cut spaces. This solves a problem of Diestel (2010, arXiv:0912.4213).
\end{abstract}

Keywords: graph theory; locally finite graph; end; homology; topological; orthogonal; cycle space; bond space; cut space

\section{Introduction}

Let $G$ be a 2-connected locally finite graph, and let $\mathcal{E}=\mathcal{E}(G)$ be its edge space over $\mathbb{F}_{2}$. We think of the elements of $\mathcal{E}$ as sets of edges, possibly infinite. Two sets of edges are orthogonal if their intersection has (finite and) even cardinality. A set $D \in \mathcal{E}$ is orthogonal to a subspace $\mathcal{F} \subseteq \mathcal{E}$ if it is orthogonal to every $F \in \mathcal{F}$. See $[4,5]$ for any definitions not given below.

The topological cycle space $\mathcal{C}_{\text {top }}(G)$ of $G$ is the subspace of $\mathcal{E}(G)$ generated (via thin sums, possibly infinite) by the circuits of $G$, the edge sets of the topological circles in the Freudenthal compactification $|G|$ of $G$. This space $\mathcal{C}_{\text {top }}(G)$ contains precisely the elements of $\mathcal{E}$ that are orthogonal to $\mathcal{B}_{\text {fin }}(G)$, the finite-cut space of $G$ [4]. The algebraic cycle space $\mathcal{C}_{\text {alg }}(G)$ of $G$ is the subspace of $\mathcal{E}$ consisting of the edge sets inducing even degrees at all the vertices. It contains precisely the elements of $\mathcal{E}$ that are orthogonal to the skew cut space $\mathcal{B}_{\text {skew }}(G)[3]$, the subspace of $\mathcal{E}$ consisting of all the cuts of $G$ with one side finite. 
The finite-cycle space $\mathcal{C}_{\text {fin }}(G)$ is the subspace of $\mathcal{E}$ generated (via finite sums) by the finite circuits of $G$. This space $\mathcal{C}_{\text {fin }}(G)$ contains precisely the elements of $\mathcal{E}$ that are orthogonal to $\mathcal{B}(G)$, the cut space of $G[4,5]$. Thus,

$$
\mathcal{C}_{\text {top }}=\mathcal{B}_{\text {fin }}^{\perp}, \quad \mathcal{C}_{\text {alg }}=\mathcal{B}_{\text {skew }}^{\perp}, \quad \mathcal{C}_{\text {fin }}=\mathcal{B}^{\perp} .
$$

Conversely,

$$
\mathcal{C}_{\text {top }}^{\perp}=\mathcal{B}_{\text {fin }}, \quad \mathcal{C}_{\text {alg }}^{\perp}=\mathcal{B}_{\text {skew }}, \quad \mathcal{C}_{\text {fin }}^{\perp}=\mathcal{B}
$$

Thus, for any of the six spaces $\mathcal{F}$ just mentioned, we have $\mathcal{F}^{\perp \perp}=\mathcal{F}$.

Proofs of most of the above six identities were first published by Casteels and Richter [3], in a more general setting. Any remaining proofs can be found in [5], except for the inclusion $\mathcal{C}_{\text {alg }}^{\perp} \supseteq \mathcal{B}_{\text {skew }}$, which is easy.

The six subspaces of $\mathcal{E}$ mentioned above are the ones most relevant to the homology of locally finite infinite graphs. See [5], Diestel and Sprüssel [6], and Georgakopoulos [7, 8]. Our aim in this note is to facilitate orthogonality proofs for these spaces by showing that, whenever $\mathcal{F}$ is one of them, a set $D$ of edges is orthogonal to $\mathcal{F}$ as soon as it is orthogonal to the minimal nonzero elements of $\mathcal{F}$.

This is easy when $\mathcal{F}$ is $\mathcal{C}_{\text {fin }}$ or $\mathcal{B}_{\text {fin }}$ or $\mathcal{B}_{\text {skew }}$ :

Proposition 1. Let $\mathcal{F}$ be a subspace of $\mathcal{E}$ all of whose elements are finite sets of edges. Then $\mathcal{F}$ is generated (via finite sums) by its $\subseteq$-minimal nonzero elements.

Proof. For a contradiction suppose that some $F \in \mathcal{F}$ is not a finite sum of finitely many minimal nonzero elements of $\mathcal{F}$. Choose $F$ with $|F|$ minimal. As $F$ is not minimal itself, by assumption, it properly contains a minimal nonzero element $F^{\prime}$ of $\mathcal{F}$. As $F$ is finite, $F+F^{\prime}=F \backslash F^{\prime} \in \mathcal{F}$ has fewer elements than $F$, so there is a finite family $\left(M_{i}\right)_{i \leqslant n}$ of minimal nonzero elements of $\mathcal{F}$ with $\sum_{i \leqslant n} M_{i}=F+F^{\prime}$. This contradicts our assumption, as $F^{\prime}+\sum_{i \leqslant n} M_{i}=F$.

Corollary 2. If $\mathcal{F} \in\left\{\mathcal{C}_{\text {fin }}, \mathcal{B}_{\text {fin }}, \mathcal{B}_{\text {skew }}\right\}$, a set $D$ of edges is orthogonal to $\mathcal{F}$ as soon as $D$ is orthogonal to all the minimal nonzero elements of $\mathcal{F}$.

When $\mathcal{F} \in\left\{\mathcal{C}_{\text {top }}, \mathcal{C}_{\text {alg }}, \mathcal{B}\right\}$, the statement of Corollary 2 is generally false for graphs that are not 3-connected. Here are some examples.

For $\mathcal{F}=\mathcal{B}$, let $G$ be the graph obtained from the $\mathbb{N} \times \mathbb{Z}$ grid by doubling every edge between two vertices of degree 3 and subdividing all the new edges. The set $D$ of the edges that lie in a $K^{3}$ of $G$ is orthogonal to every bond $F$ of $G$ : their intersection $D \cap F$ is finite and even. But $D$ is not orthogonal to every element of $\mathcal{F}=\mathcal{B}$, since it meets some cuts that are not bonds infinitely.

For $\mathcal{F}=\mathcal{C}_{\text {top }}$, let $B$ be an infinite bond of the infinite ladder $H$, and let $G$ be the graph obtained from $H$ by subdividing every edge in $B$. Then the set $D$ of edges that are incident with subdivision vertices has a finite and even intersection with every topological circuit $C$, finite or infinite, but it is not orthogonal to every element of $\mathcal{C}_{\text {top }}$, since it meets some of them infinitely. 
For $\mathcal{F}=\mathcal{C}_{\text {alg }}$ we can re-use the example just given for $\mathcal{C}_{\text {top }}$, since for 1-ended graphs like the ladder the two spaces coincide.

However, if $G$ is 3 -connected, an edge set is orthogonal to every element of $\mathcal{C}_{\text {top }}, \mathcal{C}_{\text {alg }}$ or $\mathcal{B}$ as soon as it is orthogonal to every minimal nonzero element:

Theorem 3. Let $G=(V, E)$ be a locally finite 3-connected graph, and $F, D \subseteq E$.

(i) $F \in \mathcal{C}_{\text {top }}^{\perp}$ as soon as $F$ is orthogonal to all the minimal nonzero elements of $\mathcal{C}_{\text {top }}$, the topological circuits of $G$.

(ii) $F \in \mathcal{C}_{\text {alg }}^{\perp}$ as soon as $F$ is orthogonal to all the minimal nonzero elements of $\mathcal{C}_{\text {alg }}$, the finite circuits and the edge sets of double rays in $G$.

(iii) $D \in \mathcal{B}^{\perp}$ as soon as $D$ is orthogonal to all the minimal nonzero elements of $\mathcal{B}$, the bonds of $G$.

Although Theorem 3 fails if we replace the assumption of 3 -connectedness with 2connectedness, it turns out that we need a little less than 3-connectedness. Recall that an end $\omega$ of $G$ has (combinatorial) vertex-degree $k$ if $k$ is the maximum number of vertexdisjoint rays in $\omega$. Halin [9] showed that every end in a $k$-connected locally finite graph has vertex-degree at least $k$. Let us call an end $\omega$ of $G k$-padded if for every ray $R \in \omega$ there is a neighbourhood $U$ of $\omega$ such that for every vertex $u \in U$ there is a $k$-fan from $u$ to $R$ in $G$, a subdivided $k$-star with centre $u$ and leaves on $R .^{1}$ If every end of $G$ is $k$-padded, we say that $G$ is $k$-padded at infinity. Note that $k$-connected graphs are $k$-padded at infinity. Our proof of Theorem 3(i) and (ii) will use only that every end has vertex-degree at least 3 and that $G$ is 2-connected. Similarly, and in a sense dually, our proof of Theorem 3(iii) uses only that every end has vertex-degree at least 2 and $G$ is 3-padded at infinity.

Theorem 4. Let $G=(V, E)$ be a locally finite 2-connected graph.

(i) If every end of $G$ has vertex-degree at least 3 , then $F \in \mathcal{C}_{\text {top }}^{\perp}$ as soon as $F$ is orthogonal to all the minimal nonzero elements of $\mathcal{C}_{\text {top }}$, the topological circuits of $G$.

(ii) If every end of $G$ has vertex-degree at least 3 , then $F \in \mathcal{C}_{\text {alg }}^{\perp}$ as soon as $F$ is orthogonal to all the minimal nonzero elements of $\mathcal{C}_{\text {alg }}$, the finite circuits and the edge sets of double rays in $G$.

(iii) If $G$ is 3-padded at infinity, then $D \in \mathcal{B}^{\perp}$ as soon as $D$ is orthogonal to all the minimal nonzero elements of $\mathcal{B}$, the bonds of $G$.

In general, our notation follows [4]. In particular, given an end $\omega$ in a graph $G$ and a finite set $S \subseteq V(G)$ of vertices, we write $C(S, \omega)$ for the unique component of $G-S$ that contains a ray $R \in \omega$. The vertex-degree of $\omega$ is the maximum number of vertex-disjoint rays in $\omega$. The mathematical background required for this paper is covered in $[5,6]$. For earlier results on the cycle and cut space see Bruhn and Stein $[1,2]$.

\footnotetext{
${ }^{1}$ For example, if $G$ is the union of complete graphs $K_{1}, K_{2}, \ldots$ with $\left|K_{i}\right|=i$, each meeting the next in exactly one vertex (and these are all distinct), then the unique end of $G$ is $k$-padded for every $k \in \mathbb{N}$.
} 


\section{Finding disjoint paths and fans}

Menger's theorem that the smallest cardinality of an $A-B$ separator in a finite graph is equal to the largest cardinality of a set of disjoint $A-B$ paths trivially extends to infinite graphs. Thus in a locally finite $k$-connected graph, there are $k$ internally disjoint paths between any two vertices. In Lemmas 5 and 6 we show that, for two such vertices that are close to an end $\omega$, these connecting paths need not use vertices too far away from $\omega$.

In a graph $G$ with vertex sets $X, Y \subseteq V(G)$ and vertices $x, y \in V(G)$, a $k$-fan from $X$ (or $x$ ) to $Y$ is a subdivided $k$-star whose center lies in $X$ (or is $x$ ) and whose leaves lie in $Y$. A $k$-linkage from $x$ to $y$ is a union of $k$ internally disjoint $x-y$ paths. We may refer to a sequence $\left(v_{i}\right)_{i \in \mathbb{N}}$ simply by $\left(v_{i}\right)$, and use $\bigcup\left(v_{i}\right):=\bigcup_{i \in \mathbb{N}}\left\{v_{i}\right\}$ for brevity.

Lemma 5. Let $G$ be a locally finite graph with an end $\omega$, and let $\left(v_{i}\right)_{i \in \mathbb{N}}$ and $\left(w_{i}\right)_{i \in \mathbb{N}}$ be two sequences of vertices converging to $\omega$. Let $k$ be a positive integer.

(i) If for infinitely many $n \in \mathbb{N}$ there is a $k$-fan from $v_{n}$ to $\bigcup\left(w_{i}\right)$, then there are infinitely many disjoint such $k$-fans.

(ii) If for infinitely many $n \in \mathbb{N}$ there is a k-linkage from $v_{n}$ to $w_{n}$, then there are infinitely many disjoint such $k$-linkages.

Proof. For a contradiction, suppose $k \in \mathbb{N}$ is minimal such that there is a locally finite graph $G=(V, E)$ with sequences $\left(v_{i}\right)_{i \in \mathbb{N}}$ and $\left(w_{i}\right)_{i \in \mathbb{N}}$ in which either (i) or (ii) fails. Then $k>1$, since for every finite set $S \subseteq V(G)$ the unique component $C(S, \omega)$ of $G-S$ that contains rays from $\omega$ is connected and contains all but finitely many vertices from $\bigcup\left(v_{i}\right)$ and $\bigcup\left(w_{i}\right)$.

For a proof of (i) it suffices to show that for every finite set $S \subseteq V(G)$ there is an integer $n \in \mathbb{N}$ and a $k$-fan from $v_{n}$ to $\bigcup\left(w_{i}\right)$ avoiding $S$. Suppose there is a finite set $S \subseteq V(G)$ that meets all $k$-fans from $\bigcup\left(v_{i}\right)$ to $\bigcup\left(w_{i}\right)$. By the minimality of $k$, there are infinitely many disjoint $(k-1)$-fans from $\bigcup\left(v_{i}\right)$ to $\bigcup\left(w_{i}\right)$ in $C:=C(S, \omega)$. Thus, there is a subsequence $\left(v_{i}^{\prime}\right)_{i \in \mathbb{N}}$ of $\left(v_{i}\right)_{i \in \mathbb{N}}$ in $C$ and pairwise disjoint $(k-1)$-fans $F_{i} \subseteq C$ from $v_{i}^{\prime}$ to $\bigcup\left(w_{i}\right)$ for all $i \in \mathbb{N}$. For every $i \in \mathbb{N}$ there is by Menger's theorem a $(k-1)$-separator $S_{i}$ separating $v_{i}^{\prime}$ from $\bigcup\left(w_{i}\right)$ in $C$, as by assumption there is no $k$-fan from $v_{i}^{\prime}$ to $\bigcup\left(w_{i}\right)$ in $C$. Let $C_{i}$ be the component of $G-\left(S \cup S_{i}\right)$ containing $v_{i}^{\prime}$.

Since $F_{i}$ is a subdivided $\left|S_{i}\right|$-star, $S_{i} \subseteq V\left(F_{i}\right)$. Hence for all $i \neq j$, our assumption of $F_{i} \cap F_{j}=\emptyset$ implies that $F_{i} \cap S_{j}=\emptyset$, and hence that $F_{i} \cap C_{j}=\emptyset$. But then also $C_{i} \cap C_{j}=\emptyset$, since any vertex in $C_{i} \cap C_{j}$ could be joined to $v_{j}^{\prime}$ by a path $P$ in $C_{j}$ and to $v_{i}^{\prime}$ by a path $Q$ in $C_{i}$, giving rise to a $v_{j}^{\prime}-\bigcup\left(w_{i}\right)$ path in $P \cup Q \cup F_{i}$ avoiding $S_{j}$, a contradiction.

As $S \cup S_{i}$ separates $v_{i}^{\prime}$ from $\bigcup\left(w_{i}\right)$ in $G$ and there is, by assumption, a $k$-fan from $v_{i}^{\prime}$ to $\bigcup\left(w_{i}\right)$ in $G$, there are at least $k$ distinct neighbours of $C_{i}$ in $S \cup S_{i}$. Since $\left|S_{i}\right|=k-1$, one of these lies in $S$. This holds for all $i \in \mathbb{N}$. As $C_{i} \cap C_{j}=\emptyset$ for distinct $i$ and $j$, this contradicts our assumption that $G$ is locally finite and $S$ is finite. This completes the proof of (i).

For (ii) it suffices to show that for every finite set $S \subseteq V(G)$ there is an integer $n \in \mathbb{N}$ such that there is a $k$-linkage from $v_{n}$ to $w_{n}$ avoiding $S$. Suppose there is a finite 
set $S \subseteq V(G)$ that meets all $k$-linkages from $v_{i}$ to $w_{i}$ for all $i \in \mathbb{N}$. By the minimality of $k$ there is an infinite family $\left(L_{i}\right)_{i \in I}$ of disjoint $(k-1)$-linkages $L_{i}$ in $C:=C(S, \omega)$ from $v_{i}$ to $w_{i}$. As earlier, there are pairwise disjoint $(k-1)$-sets $S_{i} \subseteq V\left(L_{i}\right)$ separating $v_{i}$ from $w_{i}$ in $C$, for all $i \in I$. Let $C_{i}, D_{i}$ be the components of $C-S_{i}$ containing $v_{i}$ and $w_{i}$, respectively. For no $i \in I$ can both $C_{i}$ and $D_{i}$ have $\omega$ in their closure, as they are separated by the finite set $S \cup S_{i}$. Thus for every $i \in I$ one of $C_{i}$ or $D_{i}$ contains at most finitely many vertices from $\bigcup_{i \in I} L_{i}$. By symmetry, and replacing $I$ with an infinite subset of itself if necessary, we may assume the following:

The components $C_{i}$ with $i \in I$ each contain only finitely many vertices from $\bigcup_{i \in I} L_{i}$.

If infinitely many of the components $C_{i}$ are pairwise disjoint, then $S$ has infinitely many neighbours as earlier, a contradiction. By Ramsey's theorem, we may thus assume that

$$
C_{i} \cap C_{j} \neq \emptyset \text { for all } i, j \in I \text {. }
$$

Note that if $C_{i}$ meets $L_{j}$ for some $j \neq i$, then $C_{i} \supseteq L_{j}$, since $L_{j}$ is disjoint from $L_{i} \supseteq S_{i}$. By (1), this happens for only finitely many $j>i$. We can therefore choose an infinite subset of $I$ such that $C_{i} \cap L_{j}=\emptyset$ for all $i<j$ in $I$. In particular, $\left(C_{i} \cup S_{i}\right) \cap S_{j}=\emptyset$ for $i<j$. By (2), this implies that

$$
C_{i} \cup S_{i} \subseteq C_{j} \text { for all } i<j \text {. }
$$

By assumption, there exists for each $i \in I$ some $v_{i}-w_{i}$ linkage of $k$ independent paths in $G$, one of which avoids $S_{i}$ and therefore meets $S$. Let $P_{i}$ denote its final segment from its last vertex in $S$ to $w_{i}$. As $w_{i} \in C \backslash\left(C_{i} \cup S_{i}\right)$ and $P_{i}$ avoids both $S_{i}$ and $S$ (after its starting vertex in $S$ ), we also have

$$
P_{i} \cap C_{i}=\emptyset
$$

On the other hand, $L_{i}$ contains $v_{i} \in C_{i} \subseteq C_{i+1}$ and avoids $S_{i+1}$, so $w_{i} \in L_{i} \subseteq C_{i+1}$. Hence $P_{i}$ meets $S_{j}$ for every $j \geqslant i+1$ such that $P_{i} \nsubseteq S \cup C_{j}$. Since the $L_{j} \supseteq S_{j}$ are disjoint for different $j$, this happens for only finitely many $j>i$. Deleting those $j$ from $I$, and repeating that argument for increasing $i$ in turn, we may thus assume that $P_{i} \subseteq S \cup C_{i+1}$ for all $i \in I$. By (3) and (4) we deduce that $P_{i} \backslash S$ are now disjoint for different values of $i \in I$. Hence $S$ contains a vertex of infinite degree, a contradiction.

Recall that $G$ is $k$-padded at an end $\omega$ if for every ray $R \in \omega$ there is a neighbourhood $U$ such that for all vertices $u \in U$ there is a $k$-fan from $u$ to $R$ in $G$. Our next lemma shows that, if we are willing to make $U$ smaller, we can find the fans locally around $\omega$ :

Lemma 6. Let $G$ be a locally finite graph with a $k$-padded end $\omega$. For every ray $R \in \omega$ and every finite set $S \subseteq V(G)$ there is a neighbourhood $U \subseteq C(S, \omega)$ of $\omega$ such that from every vertex $u \in U$ there is a $k$-fan in $C(S, \omega)$ to $R$. 
Proof. Suppose that, for some $R \in \omega$ and finite $S \subseteq V(G)$, every neighbourhood $U \subseteq$ $C(S, \omega)$ of $\omega$ contains a vertex $u$ such that $C(S, \omega)$ contains no $k$-fan from $u$ to $R$. Then there is a sequence $u_{1}, u_{2}, \ldots$ of such vertices converging to $\omega$. As $\omega$ is $k$-padded there are $k$-fans from infinitely many $u_{i}$ to $R$ in $G$. By Lemma 5(i) we may assume that these fans are disjoint. By the choice of $u_{1}, u_{2}, \ldots$, all these disjoint fans meet the finite set $S$, a contradiction.

\section{The proof of Theorems 3 and 4}

As pointed out in the introduction, Theorem 4 implies Theorem 3. It thus suffices to prove Theorem 4 , of which we prove (i) first. Consider a set $F \neq \emptyset$ of edges that meets every circuit of $G$ evenly. We have to show that $F \in \mathcal{C}_{\text {top }}^{\perp}$, i.e., that $F$ is a finite cut. (Recall that $\mathcal{C}_{\text {top }}^{\perp}$ is known to equal $\mathcal{B}_{\text {fin }}$, the finite-cut space [5].) As $F$ meets every finite cycle evenly it is a cut, with bipartition $(A, B)$ say. Suppose $F$ is infinite. Let $\mathcal{R}$ be a set of three disjoint rays that belong to an end $\omega$ in the closure of $F$. Every $R-R^{\prime}$ path $P$ for two distinct $R, R^{\prime} \in \mathcal{R}$ lies on the unique topological circle $C\left(R, R^{\prime}, P\right)$ that is contained in $R \cup R^{\prime} \cup P \cup\{\omega\}$. As every circuit meets $F$ finitely, we deduce that no ray in $\mathcal{R}$ meets $F$ again and again. Replacing the rays in $\mathcal{R}$ with tails of themselves as necessary, we may thus assume that $F$ contains no edge from any of the rays in $\mathcal{R}$. Suppose $F$ separates $\mathcal{R}$, with the vertices of $R \in \mathcal{R}$ in $A$ and the vertices of $R^{\prime}, R^{\prime \prime} \in \mathcal{R}$ in $B$ say. Then there are infinitely many disjoint $R-\left(R^{\prime} \cup R^{\prime \prime}\right)$ paths each meeting $F$ at least once. Infinitely many of these disjoint paths avoid one of the rays in $B$, say $R^{\prime \prime}$. The union of these paths together with $R$ and $R^{\prime}$ contains a ray $W \in \omega$ that meets $F$ infinitely often. For every $R^{\prime \prime}-W$ path $P$, the circle $C\left(W, R^{\prime \prime}, P\right)$ meets $F$ in infinitely many edges, a contradiction. Thus we may assume that $F$ does not separate $\mathcal{R}$, and that $G[A]$ contains $\bigcup \mathcal{R}$.

As $\omega$ lies in the closure of $F$, there is a sequence $\left(v_{i}\right)_{i \in \mathbb{N}}$ of vertices in $B$ converging to $\omega$. As $G$ is 2 -connected there is a 2 -fan from each $v_{i}$ to $\bigcup \mathcal{R}$ in $G$. By Lemma 5 there are infinitely many disjoint 2 -fans from $\bigcup\left(v_{i}\right)$ to $\bigcup \mathcal{R}$. We may assume that every such fan has at most two vertices in $\bigcup \mathcal{R}$. Then infinitely many of these fans avoid some fixed ray in $\mathcal{R}$, say $R$. The two other rays plus the infinitely many 2 -fans meeting only these together contain a ray $W \in \omega$ that meets $F$ infinitely often and is disjoint from $R$. Then for every $R-W$ path $P$ we get a contradiction, as $C(R, W, P)$ is a circle meeting $F$ in infinitely many edges.

For a proof of (ii), note first that the minimal elements of $\mathcal{C}_{\text {alg }}$ are indeed the finite circuits and the edge sets of double rays in $G$. Indeed, these are clearly in $\mathcal{C}_{\text {alg }}$ and minimal. Conversely, given any element of $\mathcal{C}_{\text {alg }}$, a set $D$ of edges inducing even degrees at all the vertices, we can greedily find for any given edge $e \in D$ a finite circuit or double ray with all its edges in $D$ that contains $e$. We may thus decompose $D$ inductively into disjoint finite circuits and edge sets of double rays, since deleting finitely many such sets from $D$ clearly produces another element of $\mathcal{C}_{\text {alg }}$, and including in each circuit or double ray chosen the smallest undeleted edge in some fixed enumeration of $D$ ensures that the entire set $D$ is decomposed. If $D$ is minimal in $\mathcal{C}_{\text {alg }}$, it must therefore itself be a finite circuit or the edge set of a double ray. 
Consider a set $F$ of edges that fails to meet some set $D \in \mathcal{C}_{\text {alg }}$ evenly; we have to show that $F$ also fails to meet some finite circuit or double ray evenly. If $|F \cap D|$ is odd, then this follows from our decomposition of $D$ into disjoint finite circuits and edges sets of double rays. We thus assume that $F \cap D$ is infinite. Since $|G|$ is compact, we can find a sequence $e_{1}, e_{2}, \ldots$ of edges in $F \cap D$ that converges to some end $\omega$. Let $R_{1}, R_{2}, R_{3}$ be disjoint rays in $\omega$, which exist by our assumption that $\omega$ has vertex-degree at least 3 . Subdividing each edge $e_{i}$ by a new vertex $v_{i}$, and using that $G$ is 2-connected, we can find for every $i$ a 2-fan from $v_{i}$ to $W=V\left(R_{1} \cup R_{2} \cup R_{3}\right)$ that has only its last vertices and possibly $v_{i}$ in $W$. By Lemma 5 , with $w_{1}, w_{2}, \ldots$ an enumeration of $W$, some infinitely many of these fans are disjoint. Renaming the rays $R_{i}$ and replacing $e_{1}, e_{2}, \ldots$ with a subsequence as necessary, we may assume that either all these fans have both endvertices on $R_{1}$, or that they all have one endvertex on $R_{1}$ and the other on $R_{2}$. In both cases all these fans avoid $R_{3}$, so we can find a ray $R$ in the union of $R_{1}, R_{2}$ and these fans (suppressing the subdividing vertices $v_{i}$ again) that contains infinitely many $e_{i}$ and avoids $R_{3}$. Linking $R$ to a tail of $R_{3}$ we thus obtain a double ray in $G$ that contains infinitely many $e_{i}$, as desired.

To prove (iii), let $D \subseteq E$ be a set of edges that meets every bond evenly. We have to show that $D \in \mathcal{B}^{\perp}$, i.e., that $D$ has an (only finite and) even number of edges also in every cut that is not a bond.

As $D$ meets every finite bond evenly, and hence every finite cut, it lies in $\mathcal{B}_{\text {fin }}^{\perp}=\mathcal{C}_{\text {top }}$. We claim that

$D$ is a disjoint union of finite circuits.

To prove $(\star)$, let us show first that every edge $e \in D$ lies in some finite circuit $C \subseteq D$. If not, the endvertices $u, v$ of $e$ lie in different components of $(V, D \backslash\{e\})$, and we can partition $V$ into two sets $A, B$ so that $e$ is the only $A-B$ edge in $D$. The cut of $G$ of all its $A-B$ edges is a disjoint union of bonds [4], one of which meets $D$ in precisely $e$. This contradicts our assumption that $D$ meets every bond of $G$ evenly.

For our proof of $(\star)$, we start by enumerating $D$, say as $D=:\left\{e_{1}, e_{2}, \ldots\right\}=: D_{0}$. Let $C_{0} \subseteq D_{0}$ be a finite circuit containing $e_{0}$, let $D_{1}:=D_{0} \backslash C_{0}$, and notice that $D_{1}$, like $D_{0}$, meets every bond of $G$ evenly (because $C_{0}$ does). As before, $D_{1}$ contains a finite circuit $C_{1}$ containing the edge $e_{i}$ with $i=\min \left\{j \mid e_{j} \in D_{1}\right\}$. Continuing in this way we find the desired decomposition $D=C_{1} \cup C_{2} \cup \ldots$ of $D$ into finite circuits. This completes the proof of $(\star)$.

As every finite circuit lies in $\mathcal{B}^{\perp}$, it suffices by $(\star)$ to show that $D$ is finite. Suppose $D$ is infinite, and let $\omega$ be an end of $G$ in its closure. Let us say that two rays $R$ and $R^{\prime} h u g$ $D$ if every neighbourhood $U$ of $\omega$ contains a finite circuit $C \subseteq D$ that is neither separated from $R$ by $R^{\prime}$ nor from $R^{\prime}$ by $R$ in $U$. We shall construct two rays $R$ and $R^{\prime}$ that hug $D$, inductively as follows.

Let $S_{0}=\emptyset$, and let $R_{0}, R_{0}^{\prime}$ be disjoint rays in $\omega$. (These exist as $G$ is 2-connected [9].) For step $j \geqslant 1$, assume that $S_{i}, R_{i}$, and $R_{i}^{\prime}$ have been defined for all $i<j$ so that $R_{i}$ and $R_{i}^{\prime}$ each meet $S_{i}$ in precisely some initial segment (and otherwise lie in $C\left(S_{i}, \omega\right)$ ) and $S_{i}$ contains the $i$ th vertex in some fixed enumeration of $V$. Add the $j$ th vertex in this enumeration to $S_{j-1}$ and, if it lies on $R_{j-1}$ or $R_{j-1}^{\prime}$, also add the initial segment of that 
ray up to it to $S_{j-1}$. Keep calling the enlarged set $S_{j-1}$. For the following choice of $S$ we apply Lemma 6 to $S_{j-1}$ and each of $R_{j-1}$ and $R_{j-1}^{\prime}$. Let $U$ be a neighbourhood of $\omega$ such that from every vertex $v$ in $U$ there are 3 -fans in $C\left(S_{j-1}, \omega\right)$ both to $R_{j-1}$ and to $R_{j-1}^{\prime}$. Let $S \supseteq S_{j-1}$ be a finite set such that $C(S, \omega) \subseteq U$. By $(\star)$ and the choice of $\omega$, there is a finite circuit $C_{j} \subseteq D$ in $C(S, \omega)$. Then $C_{j}$ can not be separated from $R_{j-1}$ or $R_{j-1}^{\prime}$ in $C\left(S_{j-1}, \omega\right)$ by fewer than three vertices, and thus there are three disjoint paths from $C_{j}$ to $R_{j-1} \cup R_{j-1}^{\prime}$ in $C\left(S_{j-1}, \omega\right)$.

There are now two possible cases. The first is that in $C\left(S_{j-1}, \omega\right)$ the circuit $C_{j}$ is neither separated from $R_{j-1}$ by $R_{j-1}^{\prime}$ nor from $R_{j-1}^{\prime}$ by $R_{j-1}$. This case is the preferable case. In the second case one ray separates $C_{j}$ from the other. In this case we will reroute the two rays to obtain new rays as in the first case. We shall then 'freeze' a finite set containing initial parts of these rays, as well as paths from each ray to $C_{j}$. This finite fixed set will not be changed in any later step of the construction of $R$ and $R^{\prime}$. In detail, this process is as follows.

If $C\left(S_{j-1}, \omega\right)$ contains both a $C_{j}-R_{j-1}$ path $P$ avoiding $R_{j-1}^{\prime}$ and a $C_{j}-R_{j-1}^{\prime}$ path $P^{\prime}$ avoiding $R_{j-1}$, let $Q$ and $Q^{\prime}$ be the initial segments of $R_{j-1}$ and $R_{j-1}^{\prime}$ up to $P$ and $P^{\prime}$, respectively. Then let $R_{j}=R_{j-1}$ and $R_{j}^{\prime}=R_{j-1}^{\prime}$ and

$$
S_{j}=S_{j-1} \cup V(P) \cup V\left(P^{\prime}\right) \cup V(Q) \cup V\left(Q^{\prime}\right) .
$$

This choice of $S_{j}$ ensures that the rays $R, R^{\prime}$ constructed from the $R_{i}$ and $R_{i}^{\prime}$ in the limit will not separate each other from $C_{j}$, because they will satisfy $R \cap S_{j}=R_{j} \cap S_{j}$ and $R^{\prime} \cap S_{j}=R_{j}^{\prime} \cap S_{j}$.

If the ray $R_{j-1}$ separates $C_{j}$ from $R_{j-1}^{\prime}$, let $\mathcal{P}_{j}$ be a set of three $\operatorname{disjoint} C_{j}-R_{j-1}^{\prime}$ paths avoiding $S_{j-1}$. All these paths meet $R_{j-1}$. Let $P_{1} \in \mathcal{P}_{j}$ be the path which $R_{j-1}$ meets first, and $P_{3} \in \mathcal{P}_{j}$ the one it meets last. Then $R_{j-1} \cup C_{j} \cup P_{1} \cup P_{3}$ contains a ray $R_{j}$ with initial segment $R_{j-1} \cap S_{j-1}$ that meets $C_{j}$ but is disjoint from the remaining path $P_{2} \in \mathcal{P}$ and from $R_{j-1}^{\prime}$. Let $R_{j}^{\prime}=R_{j-1}^{\prime}$, and let $S_{j}$ contain $S_{j-1}$ and all vertices of $\bigcup \mathcal{P}_{j}$, and the initial segments of $R_{j-1}$ and $R_{j-1}^{\prime}$ up to their last vertex in $\bigcup \mathcal{P}$. Note that $R_{j}$ meets $C_{j}$, and that $P_{2}$ is a $C_{j}-R_{j}^{\prime}$ path avoiding $R_{j}$.

If the ray $R_{j-1}^{\prime}$ separates $C_{j}$ from $R_{j-1}$, reverse their roles in the previous part of the construction.

The edges that lie eventually in $R_{i}$ or $R_{i}^{\prime}$ as $i \rightarrow \infty$ form two rays $R$ and $R^{\prime}$ that clearly hug $D$.

Let us show that there are two disjoint combs, with spines $R$ and $R^{\prime}$ respectively, and infinitely many disjoint finite circuits in $D$ such that each of the combs has a tooth in each of these circuits. We build these combs inductively, starting with the rays $R$ and $R^{\prime}$ and adding teeth one by one.

Let $T_{0}=R$ and $T_{0}^{\prime}=R^{\prime}$ and $S_{0}=\emptyset$. Given $j \geqslant 1$, assume that $T_{i}, T_{i}^{\prime}$ and $S_{i}$ have been defined for all $i<j$. By Lemma 6 there is a neighbourhood $U \subseteq C\left(S_{j-1}, \omega\right)$ of $\omega$ such that every vertex of $U$ sends a 3-fan to $R \cup R^{\prime}$ in $C\left(S_{j-1}, \omega\right)$. Let $S \supseteq S_{j-1}$ be a finite set with $C(S, \omega) \subseteq U$. As $R$ and $R^{\prime}$ hug $D$ there is a finite cycle $C$ in $C(S, \omega)$ with edges in $D$, and which neither of the rays $R$ or $R^{\prime}$ separates from the other. By the choice of $S$, no one vertex of $C\left(S_{j-1}, \omega\right)$ separates $C$ from $R \cup R^{\prime}$ in $C\left(S_{j-1}, \omega\right)$. Hence by Menger's 
theorem there are disjoint $\left(R \cup R^{\prime}\right)-C$ paths $P$ and $Q$ in $C\left(S_{j-1}, \omega\right)$. If $P$ starts on $R$ and $Q$ starts on $R^{\prime}$ (say), let $P^{\prime}:=Q$. Assume now that $P$ and $Q$ start on the same ray $R$ or $R^{\prime}$, say on $R$. Let $Q^{\prime}$ be a path from $R^{\prime}$ to $C \cup P \cup Q$ in $C\left(S_{j-1}, \omega\right)$ that avoids $R$. As $Q^{\prime}$ meets at most one of the paths $P$ and $Q$, we may assume it does not meet $P$. Then $Q^{\prime} \cup(Q \backslash R)$ contains an $R^{\prime}-C$ path $P^{\prime}$ disjoint from $P$ and $R$. In either case, let $T_{j}=T_{j-1} \cup P$, let $T_{j}^{\prime}=T_{j-1}^{\prime} \cup P^{\prime}$, and let $S_{j}$ consist of $S_{j-1}$, the vertices in $C \cup P \cup P^{\prime}$, and the vertices on $R$ and $R^{\prime}$ up to their last vertex in $C \cup P \cup P^{\prime}$.

The unions $T=\bigcup_{i \in \mathbb{N}} T_{i}$ and $T^{\prime}=\bigcup_{i \in \mathbb{N}} T_{i}^{\prime}$ are disjoint combs that have teeth in infinitely many common disjoint finite cycles whose edges lie in $D$. Let $A$ be the vertex set of the component of $G-T$ containing $T^{\prime}$, and let $B:=V \backslash A$. Since $T$ is connected, $E(A, B)$ is a bond, and its intersection with $D$ is infinite as every finite cycle that contains a tooth from both these combs meets $E(A, B)$ at least twice. This contradiction implies that $D$ is finite, as desired.

\section{References}

[1] H. Bruhn and M. Stein. On end degrees and infinite circuits in locally finite graphs. Combinatorica, 27:269-291, 2007.

[2] H. Bruhn and M. Stein. Duality of ends. Comb., Probab. Comput., 19:47-60, 2010.

[3] K. Casteels and B. Richter. The Bond and Cycle Spaces of an Infinite Graph. J. Graph Theory, 59(2):162-176, 2008.

[4] R. Diestel. Graph Theory. Springer, 4th edition, 2010.

[5] R. Diestel. Locally finite graphs with ends: a topological approach. Discrete Math., 310-312: 2750-2765 (310); 1423-1447 (311); 21-29 (312), 2010-11. arXiv:0912. 4213.

[6] R. Diestel and P. Sprüssel. The homology of locally finite graphs with ends. Combinatorica, 30:681-714, 2010.

[7] A. Georgakopoulos. Graph topologies induced by edge lengths. In Diestel, Hahn, and Mohar, editors, Infinite graphs: introductions, connections, surveys, volume Discrete Mathematics 311, pages 1523-1542, 2011.

[8] A. Georgakopoulos. Cycle decompositions: from graphs to continua. Advances in Mathematics, 229:935-967, 2012.

[9] R. Halin. A note on Menger's theorem for infinite locally finite graphs. Abh. Math. Sem. Univ. Hamburg, 40:111-114, 1974. 